\title{
Enhancement of Activated Sludge Dewaterability by Using Filamentous Fungi as Bioadditives
}

\author{
Miguel Ángel López Zavala * and Jorge Isidro Ramos Patlán \\ Water Center for Latin America and the Caribbean, Tecnológico de Monterrey, Av. Eugenio Garza Sada Sur \\ No. 2501, Col. Tecnológico, Monterrey C.P. 64849, NL, Mexico; isidro.ramosp@gmail.com \\ * Correspondence: miganloza@itesm.mx; Tel.: +52-81-8155-2573 (ext. 106) \\ Academic Editor: Andreas N. Angelakis \\ Received: 20 September 2016; Accepted: 10 November 2016; Published: 15 November 2016
}

\begin{abstract}
In this study, the dewaterability of activated sludge inoculated with spores of two filamentous fungi (Trichoderma reesei and Aspergillus oryzae) was evaluated. The reduction of capillary suction time (CST) and the increase of sludge solids content were used as parameters to assess the dewaterability of the sludge. Furthermore, the transformation of the extracellular polymeric substances (EPS) of the sludge during the fungal conditioning and the interaction of microbial and fungal populations were addressed. Fungal conditioning reduced the CST by $25 \%$ and increased the sludge solids content by $16 \%$, denoting the enhancement of the sludge dewaterability. The optimum fungal conditioning time was three to four days. Fungi hydrolyzed the sludge EPS, allowing the release of water from the flocs matrix. Fungal conditioning increased the fungal and microbial communities. Furthermore, the increase of the fungal population augmented the hydrolytic activity of enzymes over the sludge EPS, enhancing the water release and consequently the dewaterability of the activated sludge.
\end{abstract}

Keywords: activated sludge; Aspergillus oryzae; capillary suction time; dewaterability; extracellular polymeric substances (EPS); Trichoderma reesei

\section{Introduction}

Worldwide, the activated sludge process is the most common biological method for treating domestic wastewater. Mature technology, efficient performance and low investment are some of the most important advantages of this process. However, the main drawback of the technology is the large amount of excess sludge generated that must in turn be properly managed and disposed of. Furthermore, the costs of treatment and disposal of the sludge could represent $50 \%-60 \%$ of the total operational cost of the wastewater treatment plants (WWTP) [1,2].

As a consequence of the accelerated urbanization and industrialization experienced recently by the emergent economies of the world (including Mexico) and the implementation of more stringent environmental regulations, the increase of the wastewater treatment capacity accompanied by an excess production of sludge has been reported [3,4]. Management, treatment and disposal of huge amounts of sludge have become a serious challenge for WWTP operators and government authorities because of the high cost and environmental drawbacks of the current technology. Reliable and economic solutions are needed to reduce the volume of sludge when it is treated and/or disposed of.

A common operation to reduce the volume of sludge is the dewatering process. Dewatering is the process of removing water from sludge to reduce its volume and produce a material suitable for further processing, beneficial use, or disposal. Dewatering processes produce a cake that behaves like a semi-solid or solid material. Reducing the volume cuts subsequent solid-management costs [5]. Several techniques have been used for sludge dewatering. Some of these techniques rely on natural evaporation and percolation to dewater the solids. Mechanically assisted physical means are used 
to dewater the sludge more quickly. These means include filtration, squeezing, capillary action, and centrifugal separation and compaction. The dewatering processes commonly used include centrifuges, belt-filter presses, recessed-plate filter presses, drying beds, and lagoons [6]. Chemical dewatering methods sometimes are used to improve cake solids, the solids' capture efficiency and the quality of the dewatered cake product. The most common types of chemicals used are inorganic salts (e.g., lime and ferric chloride) and organic polymers [5].

Conventional dewatering systems frequently require a relative large capital investment and a substantial share of a facility's annual budget for operation and maintenance [5]. Furthermore, the use of chemicals for conditioning can lead to a contamination problem if the dewatering product is not disposed of properly [7]. Due to these drawbacks, recently researchers have focused their attention on biological techniques which could be more environmentally friendly and economically viable. Zheng et al. [8] reported that sludge dewaterability was greatly improved when the filamentous fungi Mucor circinelloides ZG-3 and Acidithiobacillus ferrooxidans LX5 were sequentially inoculated into sludge. Murugesan et al. [9] improved flocculation and dewaterability of chemically enhanced primary treatment sludge using a pellet-forming Penicillium sp. Others have reported the use of fungi for bioseparation of suspended solids to improve the filterability and dewaterability of domestic wastewater sludge [10-13]. Fakhru'l-Razi and Molla [14] observed a 70\% reduction in specific resistance to filtration (SRF) after a six-day treatment of sludge with Mucor hiemalis.

Although some studies have shown promising results, the number of reports on this matter is limited and more research is needed to evaluate the performance of different species of fungi, their dosages, and time of treatment with different types of sludge. Thus, the use of commercially available fungi such as Aspergillus oryzae and Trichoderma reesei was selected to conduct this study. Aspergillus oryzae is a filamentous fungus which has an ability to secrete large amounts of hydrolytic enzymes. It is widely used in the manufacture of traditional fermented soy sauce in Asia [15]. Additionally, Aspergillus oryzae is genomically well characterized and considered to be a safe organism for producing food enzymes [16]. On the other hand, Trichoderma reesei is an industrially important cellulolytic filamentous fungus and is the main industrial source of cellulases and hemicellulases used to depolymerize biomass to simple sugars which are converted to chemical intermediates and biofuels, such as ethanol [17].

Thus, the aim of this research was to evaluate the enhancement of sludge dewaterability by using the aforementioned species. Additionally, the transformation of the extracellular polymeric substances (EPS) of the sludge during the fungal conditioning and the interaction of microbial and fungal populations are discussed.

\section{Materials and Methods}

\subsection{Preparation of the Activated Sludge}

Activated sludge was taken from the secondary clarifier of a municipal wastewater treatment plant located in the city of Monterrey, Mexico. The total suspended solids and total chemical oxygen demand of a 12-day-old sludge were 13,275 and 18,430 $\mathrm{mg} / \mathrm{L}$, respectively.

\subsection{Preparation of Fungal Spores Solutions}

A. oryzae (American Type Culture Collection (ATCC): 11490) and T. reesei (ATCC: 26921) lyophilized strains were purchased from American Type Culture Collection (ATCC, Manassas, VA, USA). The lyophilized strains of both fungi were reactivated in a solid medium of potato dextrose agar (PDA, Fluka Analytical, Madrid, Spain) following the procedure described in the ATCC ${ }^{\circledR}$ Mycology Culture Guide [18]. Then, live cultures were aseptically transferred to new plates containing the PDA medium and incubated for maximum two weeks at $27^{\circ} \mathrm{C}$. To prepare the fungal spores' solutions, four plates of five-day-old fungal cultures were taken and the mycelia were washed using an aqueous solution of $0.01 \%(v / v)$ Triton X-100 (Sigma-Aldrich, Toluca, Mexico). Next, $10 \mathrm{~mL}$ solution was added 
to the mycelia of each plate and let stand per $10 \mathrm{~min}$. Then, the surface of the agar plates was gently scraped with an aseptic pipette to remove the spores from the mycelia, taking care that PDA medium was not removed, and the suspension thus obtained was collected in a $50 \mathrm{~mL}$ falcon tube. Next, a second wash of the mycelia was conducted by adding $10 \mathrm{~mL}$ Triton X-100 solution to one of the plates and then this suspension was used to wash again the other three plates. This suspension was also collected into the $50 \mathrm{~mL}$ falcon tube resulting a total volume of approximately $45 \mathrm{~mL}$ of spores' suspension. The number of spores contained in the suspension was counted using a hemocytometer (Levy Improved Nebauer, Hausser Scientific, Horsham, PA, USA) and a microscope (Leica DM750, Leica Microsystems, Wetzlar, Germany).

\subsection{Experimental Device}

Lab-scale batch experiments were conducted in $5 \mathrm{~L}$ glass beakers. A $4 \mathrm{~L}$ activated sludge was added to each beaker and temperature of the reactors was set at $25^{\circ} \mathrm{C}$ using Cimarec ${ }^{\mathrm{TM}}$ Digital Stirring Hotplates (Thermo Fisher Scientific, Monterrey, NL, Mexico).

\subsection{Evaluation of Sludge Dewaterability}

Two reactors filled with $4 \mathrm{~L}$ activated sludge were prepared. One of them was inoculated with $40 \mathrm{~mL}$ fungal spores' suspension (see Section 2.2) and the other was used as the control. Both reactors were incubated for five days under the conditions described in the Section 2.3. The concentrations of spores of T. reesei and A. oryzae were $3.08 \times 10^{6}$ and $3.14 \times 10^{6}$ spores $/ \mathrm{mL}$, respectively.

Dewaterability of the activated sludge was assessed based on two parameters, capillary suction time (CST) and the sludge solids content. To determine these parameters, sludge samples from the experimental reactors were taken every $24 \mathrm{~h}$. The capillary suction time was measured using a Triton Type 319 Multi-purpose CST meter (Triton Electronics Ltd., Dunmow, Essex, UK) and the procedure described in the section 2710G of the Standard Methods for the Examination of Water and Wastewater [19]. The CST tests were conducted at $100 \mathrm{~Pa}$ of suction pressure. The sludge solids content was estimated as the weight of the total dry solids of the cake formed in the CST cylinder divided by the total weight of the cake (solids plus water), expressed as a percentage. The total solids were determined based on the procedure described in the section 2540 B of the Standard Methods for the Examination of Water and Wastewater [19]. Additionally, changes on organic load during the fungal conditioning was monitored based on sludge and supernatant COD measurements. COD was determined based on the standard protocol described in the section 5220D of Standard Methods for the Examination of Water and Wastewater [19]. All measurements were conducted by quintuplicate.

\subsection{Extraction and Analysis of EPS}

Bound and soluble EPS were extracted from activate sludge based on the protocol described by [20]. A cation exchange resin (CER) (DOWEX $50 \times 8,20-50$ mesh, sodium form; Sigma-Aldrich, St. Louis, MO, USA) and an extraction buffer composed of $2 \mathrm{mM} \mathrm{Na}_{3} \mathrm{PO}_{4}, 4 \mathrm{mM} \mathrm{NaH}_{2} \mathrm{PO}_{4}, 9 \mathrm{mM} \mathrm{NaC1}$ and $1 \mathrm{mM} \mathrm{KCI}$ at pH 7 were used. Volatile suspended solids (VSS) of the sludge were determined and $0.5 \mathrm{~g}$ were taken and centrifuged at $4200 \mathrm{~g}$-force for $15 \mathrm{~min}$. Then $10 \mathrm{~mL}$ supernatant, containing the soluble EPS, was collected and frozen for further analysis and the remaining volume was discarded. The sludge pellets were resuspended in $30 \mathrm{~mL}$ of the extraction buffer and centrifuged at $4200 \mathrm{~g}$-force for $15 \mathrm{~min}$. The supernatant was discarded and the pellets were resuspended again in $25 \mathrm{~mL}$ of the extraction buffer and homogenized for $5 \mathrm{~min}$ by using a sonic homogenizer. The suspension was transfer to an extraction beaker and $35 \mathrm{~g}$ CER (previously washed) was added. The CER/sludge suspension was stirred for $4 \mathrm{~h}$ under darkness conditions and centrifuged at $4200 \mathrm{~g}$-force for $1 \mathrm{~min}$ to remove the CER. The supernatant was centrifuged at $4200 \mathrm{~g}$-force for $15 \mathrm{~min}$. $10 \mathrm{~mL}$ supernatant, containing the bound EPS, was collected and frozen for further analysis.

For the analysis of proteins and carbohydrates, frozen supernatant samples were thawed and filtrated using $0.22 \mu \mathrm{m}$ PTFE filters (Sigma-Aldrich, Toluca, Mexico) and stored at $20^{\circ} \mathrm{C}$. Protein content 
in the bound and soluble EPS was determined using the Lowry method modified by [21]. Bovine serum albumin (BSA), Lowry reagent and Folin-Ciocalteu reagent, all purchased from Sigma-Aldrich (St. Louis, MO, USA), were used. Protein measurements were conducted by triplicate using a spectrophotometer (Hach DR500, Düsseldorf, Germany) with absorbance readings at $750 \mathrm{~nm}$.

Carbohydrates content in the bound and soluble EPS was determined qualitative and quantitatively. Qualitative determination consisted on the identification of polysaccharides based on their molecular mass. The polysaccharide separation was conducted by HPLC-SEC using an Agilent Bio-SEC3 guard $(4.6 \mathrm{~mm} \times 50 \mathrm{~mm}$; particle size: $3 \mu \mathrm{m}$, Agilent Technologies, Richardson, TX, USA) pre-column and an Agilent Bio-SEC3 $(4.6 \mathrm{~mm} \times 150 \mathrm{~mm}$; particle size: $3 \mu \mathrm{m}$, Agilent Technologies, Richardson, TX, USA) column. Nine polysaccharides standards $(0.667 \mathrm{kDa}, 6.1 \mathrm{kDa}$, $9.6 \mathrm{kDa}, 21.1 \mathrm{kDa}, 47.1 \mathrm{kDa}, 107 \mathrm{kDa}, 194 \mathrm{kDa}, 344 \mathrm{kDa}$ and $708 \mathrm{kDa}$ ) from Pullulan Polysaccharide Calibration Kits (Agilent Technologies, Cheadle, UK) were used. The calibration curved was prepared using concentrations of $2 \mathrm{mg} / \mathrm{mL}$ of each polysaccharide. The mobile phase consisted in a $0.2 \mathrm{M}$ sodium chloride solution under an isocratic mode. The chromatographic conditions were: flowrate, $0.35 \mathrm{~mL} / \mathrm{min}$; detection temperature, $30^{\circ} \mathrm{C}$; and injection volume, $100 \mu \mathrm{L}$.

Quantitative determination consisted in the measurement of simple carbohydrates by HPLC, using maltose (Sigma-Aldrich, Tokyo, Japan) and glucose (Sigma-Aldrich, St. Louis, MO, USA) as standards. A Shodex SH-G (6.0 mm $\times 50.0 \mathrm{~mm}$; particle size: $10 \mu \mathrm{m}$; Nihon Waters, Tokyo, Japan) pre-column and Shodex SH1011 $(8.0 \mathrm{~mm} \times 300 \mathrm{~mm}$; particle size: $6 \mu \mathrm{m}$, Nihon Waters, Tokyo, Japan) column were used. The mobile phase consisted in a $5 \mathrm{mM}$ sulfuric acid solution under an isocratic mode. The chromatographic conditions were flowrate $0.65 \mathrm{~mL} / \mathrm{min}$, detection temperature $50{ }^{\circ} \mathrm{C}$ and injection sample $10 \mu \mathrm{L}$.

\subsection{Interaction between Fungi and Microbial Communities}

Microbial and fungi populations in the sludge were monitored during the fungal conditioning in order to elucidate the interaction between them. The total microbial population was determined as follows, $0.1 \mathrm{~mL}$ sludge sample was diluted into $19.9 \mathrm{~mL}$ double-distilled water (1:200 dilution) and $100 \mu \mathrm{L}$ aliquot was transferred onto surface of a pre-dried Tryptone agar plate and spread uniformly using a sterile L-shaped glass rod (streak plate method). The plates were incubated for $24 \mathrm{~h}$ at $27^{\circ} \mathrm{C}$. The Tryptone agar was prepared with $8 \mathrm{~g} / \mathrm{L} \mathrm{NaCl}$ (Promega Corporation, Fitchburg, WI, USA), $10 \mathrm{~g} / \mathrm{L}$ Tryptone (Becton, Dickinson and Company, Franklin Lakes, NY, USA) and $15 \mathrm{~g} / \mathrm{L}$ microbial agar (Sigma-Aldrich, St. Louis, MO, USA ). Using a darkfield colony counter all colonies were counted on each selected plate and expressed on CFU/mL by using Equation (1).

$$
\frac{\mathrm{CFU}}{\mathrm{mL}}=\frac{\text { Plate countxDilution factor }}{\text { Aliquot volume }}
$$

For fungi, a Sabouraud glucose agar with chloramphenicol (Fluka Analytical, Shivaji Marg, New Delhi, India) was used. $1.0 \mathrm{~mL}$ sludge sample was diluted into $19.0 \mathrm{~mL}$ pre-sterile double-distilled water to have a 1:20 dilution. Using the streak plate method, $100 \mu \mathrm{L}$ aliquot was spread onto the pre-dried agar plate by duplicate. The plates were incubated for $24 \mathrm{~h}$ at $27^{\circ} \mathrm{C}$ and all colonies counted using a darkfield colony counter. The result was expressed in CFU/mL using the Equation (1).

\section{Results and Discussion}

\subsection{Capillary Suction Time (CST)}

The empirical CST test is well established as the leading method for the determination of sludge dewaterability, despite its shortcomings including restricted possibilities to model and, therefore, predict physical processes, such as the amount of water bound by the filter paper. The CST method is easy to conduct, cost-effective and accurate [22] and was used to assess the sludge dewaterability in this study. The specific resistance to filtration (SRF) test is also commonly used to estimate sludge 
dewaterability; however, SRF is much more expensive and time-consuming compared to the CST test [23]. Figure 1 presents the results of the CST tests conducted in this study. The initial CST of sludge samples was $14.5 \mathrm{~s}$. In general, the fungal conditioning reduced the CST of the sludge. The CST reduction after three days of conditioning was $25 \%$ for both fungi. At the end of the experiments (five days), the CST reduction for A. oryzae was 27\%; meanwhile, the CST for T. reesei increased unexpectedly during the last two days. There is no clear explanation for this result, but might be associated with the greater increase of the microbial community during $T$. reesei conditioning, which limited the growth of the fungi and consequently the enhancement of the sludge dewaterability, as discussed in Section 3.5. According to the literature [22-24], a large CST usually implies a poor sludge filterability; therefore, it can be said that fungal conditioning eased the filtration of the sludge, i.e., enhanced the activated sludge dewaterability. However, to confirm this result, the effect of fungal conditioning onto the solids concentration of sludge has to be considered.

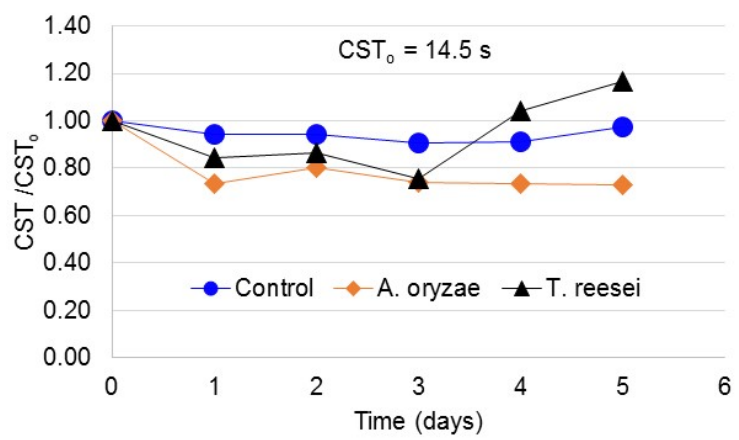

(a)

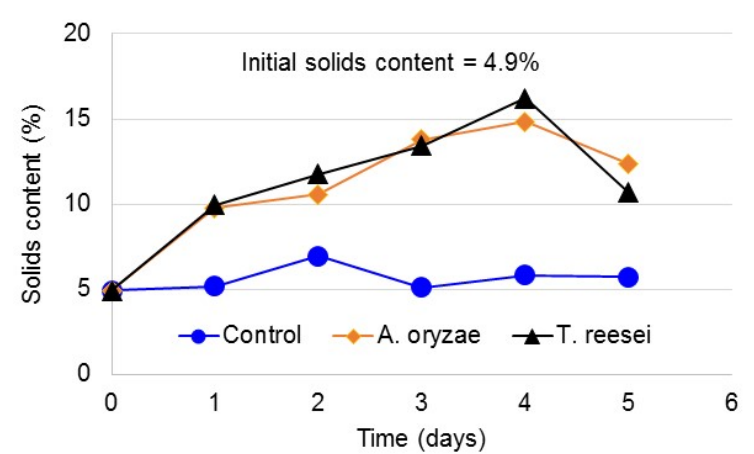

(b)

Figure 1. Effect of fungal conditioning on the activated sludge dewaterability. (a) Evolution of $\mathrm{CST} / \mathrm{CST}_{0} ;(\mathbf{b})$ Evolution of solids content.

\subsection{Sludge Solids Content}

Activated sludge dewaterability was also assessed by measuring the solids content of sludge. The results are shown in Figure 1. The initial content of solids in the raw activated sludge was in the order of $4.9 \%$. As seen, the maximum increase of solids content was achieved after four days of treatment with both fungi. For T. reesei, the maximum increase was $16.2 \%$; meanwhile, for $A$. oryzae it was $14.8 \%$. The decrease of solids content on the fifth day of conditioning might be associated with the decline of fungi populations; therefore, the greater the fungi population, the greater the increase of solids content, as discussed in Section 3.5. On the other hand, the solids content of sludge in the control did not show a significant change with respect to the initial concentration of solids; an average concentration of $5.6 \%$ was observed. Based on these results, it can be said that fungal conditioning improved activated sludge dewaterability because the solids content increased approximately $10 \%$. Moreover, these results also confirmed that the reduction of CST implied an improvement of the sludge dewaterability. However, care must be taken at the time of interpreting the CST results because the bound water content cannot be directly evaluated from the CST data [22-24].

Figure 2 depicts the transformation of the sludge during the fungal conditioning. In general, low degradation of the sludge occurred during the fungal conditioning, and total COD reductions of $14.7 \%$ for T. reesei and $8.8 \%$ for A. oryzae were observed; meanwhile, for the control, the reduction was in the order of $28.4 \%$. As seen, the particulate organic matter of sludge decreased gradually in the first four days of the fungal conditioning, while the control showed a continuous and greater decrease of particulate COD. On the other hand, the soluble organic matter (supernatant COD) increased during the first three days, especially on the control, and then the COD concentration decreased until the end of the experiments. The increase of the soluble COD is evidence of the occurrence of the hydrolysis process. The decrease of soluble COD at days 4 and 5 might be associated with the decline of the fungi 
population, as discussed in Section 3.5, which consequently caused the hydrolysis rate reduction of the extracellular polymeric substances (EPS). In case of the fungal conditioning, hydrolysis broke down the EPS from the flocs structure of the sludge, releasing water and transforming EPS into more simple molecules (soluble COD). The release of water from the flocs structure of sludge was confirmed by the increase of the solids content in the sludge cake. The increase of solids content denoted consequently better dewaterability of the activated sludge. It is well known that both fungi release hydrolytic enzymes such as $\alpha$-amylase, which is a hydrolase that catalyzes the hydrolysis of chemical bonds. Further analysis of EPS transformation will be addressed in Section 3.3. Unlike fungal conditioning, in the control, the hydrolysis of biomass occurred with minimum breakdown of EPS. This was confirmed by the almost negligible change in the solids content of the sludge which denoted no water release from the flocs structure and consequently minimum change of the activated sludge dewaterability. Likewise, the degradation observed during the experiments did not affect the sludge dewaterability in the control.

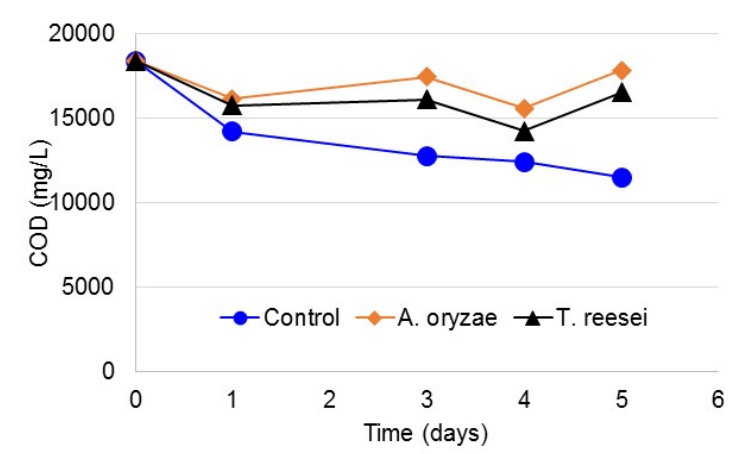

(a)

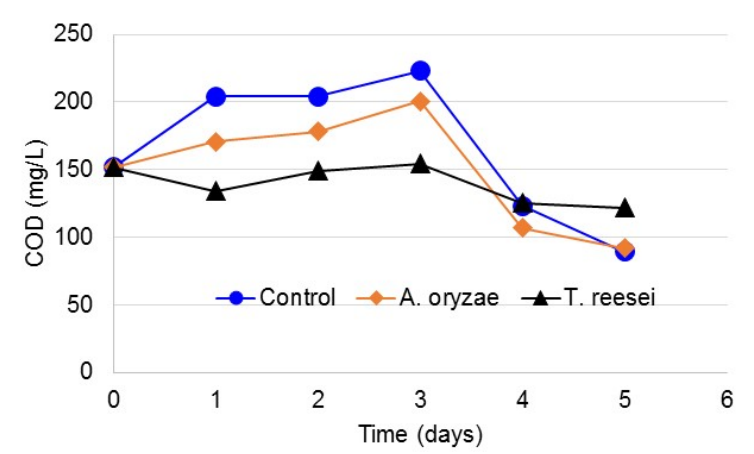

(b)

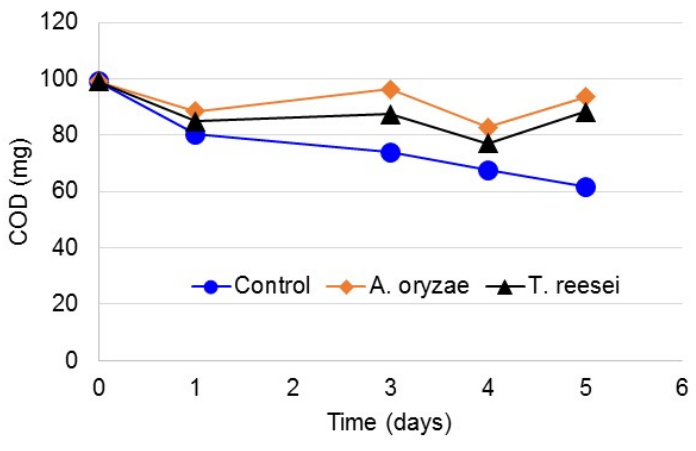

(c)

Figure 2. Evolution of COD during the fungal conditioning of activated sludge. (a) Particulate COD (biosolids); (b) soluble COD (supernatant); (c) total COD (particulate + soluble).

\subsection{Protein Content of Bound and Soluble EPS}

The results of protein analysis in bound and soluble EPS are shown in Figure 3. As seen, the concentration of proteins in bound and soluble EPS increased during the fungal conditioning in comparison with the control. The maximum concentration of proteins was observed after three days of conditioning, which was especially visible in bound EPS for T. reesei. The increase of protein contents means that proteins were released from the EPS matrix. The protein chains were broken down by extracellular hydrolytic enzymes secreted by the fungi, as reported by [25]. Secretion of the enzyme protease by the two types of fungi used in this study has been reported in the literature $[26,27]$. Regarding the results of the control, a slight increase in protein concentration was only observed in the first two days of the experiments; this means that protein chains of the EPS were hydrolyzed to a limit. 
Thus, these results confirm that fungal conditioning contributed to enhancing the hydrolysis of EPS which consequently enhanced the sludge dewaterability, as discussed in Section 2.3.

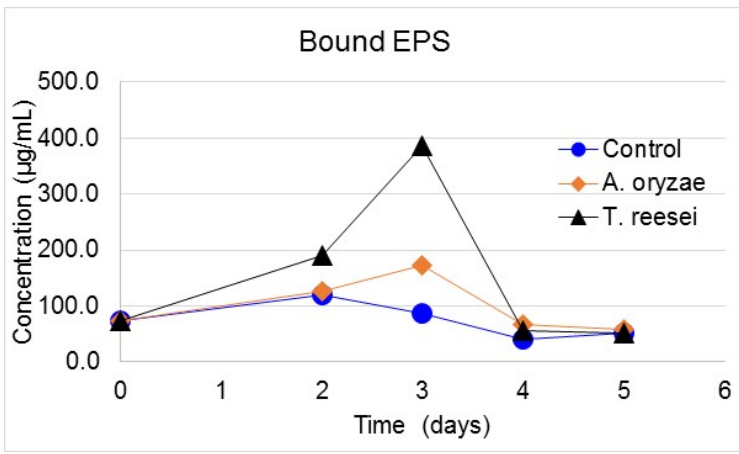

(a)

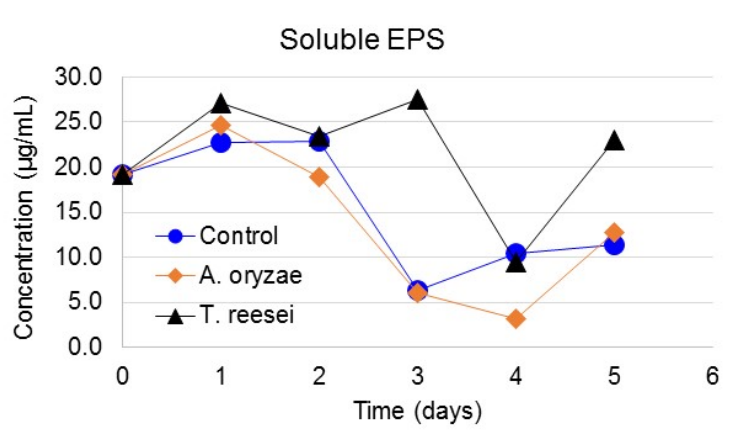

(b)

Figure 3. Protein content in bound and soluble EPS. (a) Bound EPS; (b) Soluble EPS.

\subsection{Carbohydrate Content in Bound and Soluble EPS}

The qualitative determination of polysaccharides consisted of the identification of polysaccharides based on their molecular mass. Figure 4 shows the molecular mass and the elution time for each carbohydrate standard. For better separation performance, only three molecular mass ranges were selected for analysis, 194-344, 9.6-47.1 and $0.667 \mathrm{kDa}$, with elution times of 6.0, 9.7 and $11.7 \mathrm{~min}$, respectively. Peak areas were used to estimate the two molecular mass ranges of 194-344 and 9.6-47.1 kDa, and the peak height for the molecular mass $0.667 \mathrm{kDa}$ due to distortion created by the mobile phase at elution time 11.7. In Figure 5, the results of the polysaccharide identification are shown. As seen in the case of bound EPS, polysaccharides of the three molecular mass ranges were detected and their response was greater during fungal conditioning in comparison with that of the control. The maximum polysaccharide response was observed after four days of conditioning for the larger molecular sizes and after three days for the molecular mass of $0.667 \mathrm{kDa}$. The increase of the polysaccharide response means that carbohydrates were released from the EPS matrix due to the hydrolysis conducted by the extracellular hydrolytic enzymes secreted by the fungi. This result is in agreement with that obtained for proteins. On the other hand, in the soluble EPS extracts only the $0.667 \mathrm{kDa}$ polysaccharide was detected. Its content increased with time; however, unlike the bound EPS extracts, the content was greater on the control samples. The $0.667 \mathrm{kDa}$ molecular mass corresponds to stachyose, a tetrasaccharide that is hydrolyzed by $\alpha$-galactosidase, an enzyme secreted by both fungi. Thus, when the stachyose was released from the bound EPS matrix and solubilized, it was hydrolyzed by the $\alpha$-galactosidase, decreasing its content in the soluble EPS of the fungal-conditioned samples. Upon hydrolysis, stachyose gives four molecules of the same or different monosaccharides, among them glucose.

Regarding the quantitative determination of simple carbohydrates such maltose and glucose in bound and soluble EPS, the results of the HPLC analysis showed that only traces of maltose were detected in some EPS samples of the fungal-conditioned sludge; meanwhile, glucose was found in small amounts, on the order of $\mu \mathrm{g} / \mathrm{L}$, only in the soluble EPS of the fungal-conditioned and control samples (Figure 6). Maltose may have been rapidly hydrolyzed to glucose or, simply, this carbohydrate was not a relevant product of the hydrolytic activity of the fungal enzymes. The glucose content in the fungal-conditioned samples was greater than in the control during the first three days; then, there was not a big difference between the content of all the samples. Simple carbohydrates are easily assimilated by microorganisms; therefore, as soon as glucose was available from the hydrolysis process it was consumed by the microbial and fungal communities. Consequently, a low glucose content in the EPS extracts was quantified. From these results, some evidence of the polysaccharides' hydrolysis is observed, but it is less conclusive than those of the qualitative determination. 


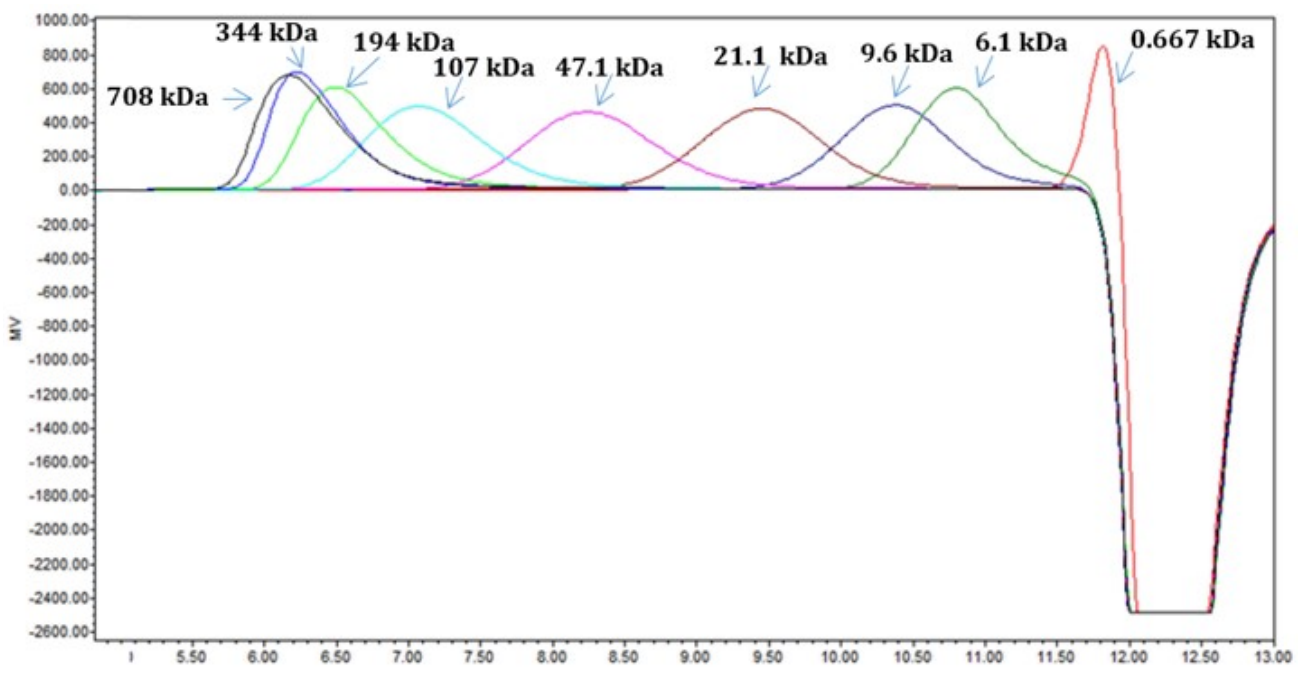

Figure 4. Identification of molecular mass and elution times of polysaccharide standards.

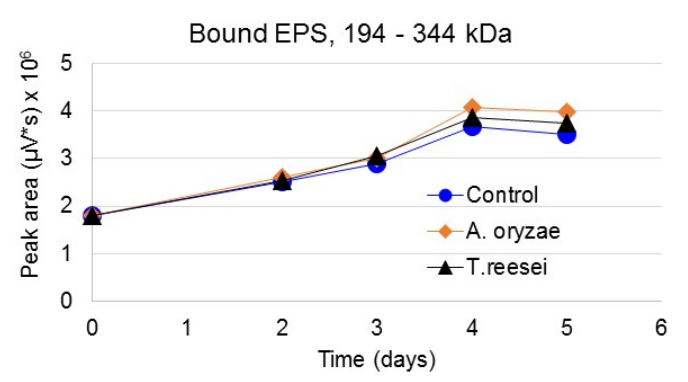

(a)

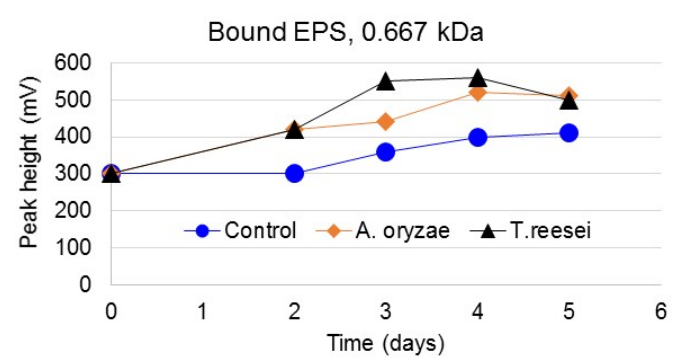

(c)

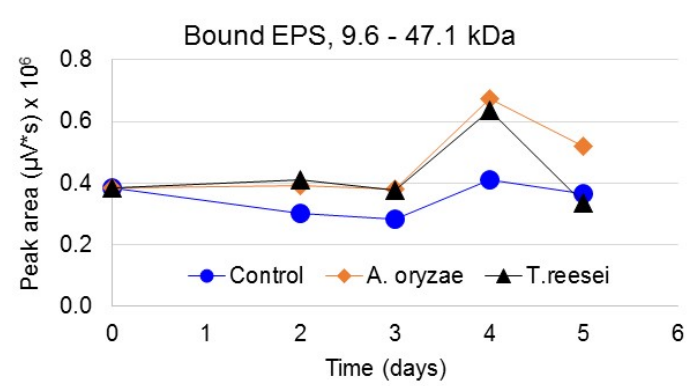

(b)

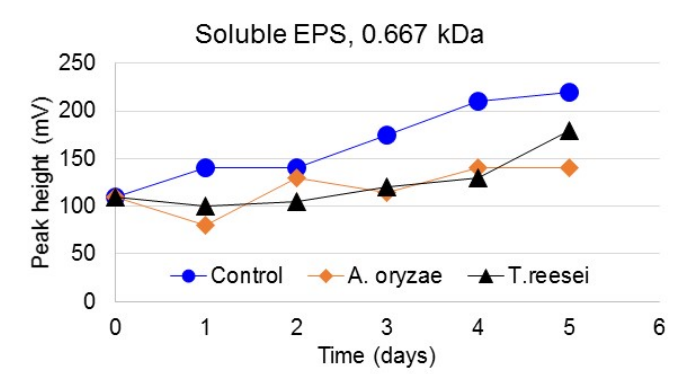

(d)

Figure 5. Qualitative determination of polysaccharides in EPS extracts. (a) bound EPS, 194-344 kDa; (b) bound EPS, 9.6-47.1 kDa; (c) bound EPS, 0.667 kDa; (d) soluble EPS, 0.667 kDa.

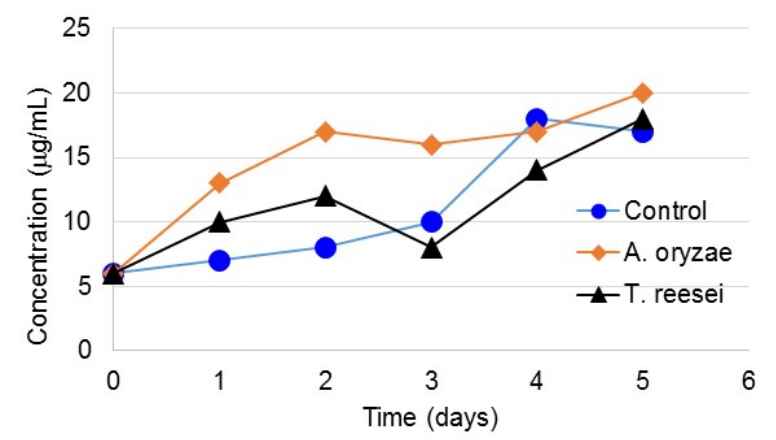

Figure 6. Content of glucose in soluble EPS extracts. 


\subsection{Interaction of Microbial and Fungi Communities}

As seen in previous results, extracellular hydrolytic enzymes secreted by fungi hydrolyzed the EPS, allowing the release of water from the sludge matrix and, consequently, improving the sludge dewaterability. Thus, the interaction of fungi with the microbial community during the conditioning of the activated sludge was determined. Figure 7 shows the behavior of the microbial and fungal communities during the fungal conditioning of sludge. As seen, the microbial community of sludge increased remarkably after the inoculation with fungi, which was especially evident in the case of T. reesei. The maximum microbial population was observed after two days of conditioning; then, the population began to decline. Hydrolysis of EPS during the fungal conditioning transformed polymeric substances into more simple molecules that were assimilated by bacteria and fungi to grow up. In the case of the control, the microbial content of the sludge remained constant during the first two days and then began to decline because no substrate was available to the microorganisms; consequently, the decaying process of the biomass began, which was confirmed by the reduction of particulate COD, as shown in Figure 2. On the other hand, it is evident that not only bacteria but also the fungal community benefited from the concomitant interaction among them. As seen in Figure 7, the fungal population increased rapidly, reaching the maximum just one day after the inoculation; then, it began to decline, returning to the initial concentration at the end of the experiments. A. oryzae showed the greatest growth, but this growth impacted negatively on the total microbial community because lower growth was observed in comparison with T. reesei. The decrease of the microbial and fungi populations after reaching a maximum could be associated with the release of water from the sludge matrix that may limit the hydrolytic activity of the fungal enzymes which consequently limit the substrate availability. Another reason could be the competition between bacteria and fungi for substrate which could limit the continuous growth of the fungal community and, consequently, their hydrolytic capacity. From these results one fact is clear: fungal conditioning had a positive effect on the microbial community. Furthermore, the increase of the fungal population augmented the hydrolytic activity of enzymes over the sludge EPS, enhancing the water release and, consequently, the dewaterability of the activated sludge.

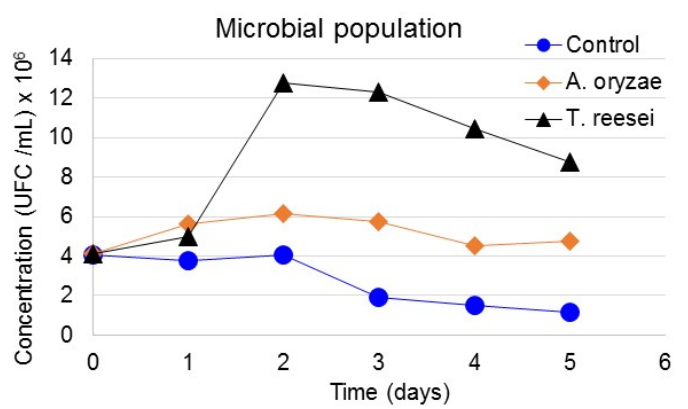

(a)

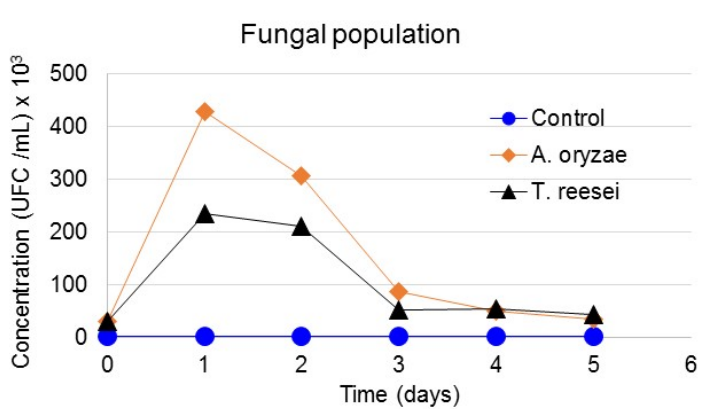

(b)

Figure 7. Interaction of microbial and fungal populations during the fungal conditioning. (a) microbial population; (b) fungal population.

Regarding the full-scale implementation of the proposed process of fungi conditioning, some conventional operations such as thickening, chemical conditioning and dewatering in conventional municipal wastewater treatment plants can be improved by adding any of the two fungi used in this study. It was found that the optimum fungal conditioning time was three to four days, achieving $16 \%$ sludge solids content; however, at one and two days the contents obtained were $10 \%$ and $12 \%$, respectively. These values are greater than those obtained for conventional thickening processes $(2 \%-3 \%$ [6]) of activated sludge for retention times up to one to two days; therefore, the method proposed in this study can practically replace conventional thickening, using the same retention time 
(one to two days), or enhance it by the addition of fungi, increasing even the retention time to three to four days. Furthermore, if anaerobic digestion of the sludge is conducted, its efficiency could be improved due to the sludge EPS being hydrolyzed during the fungal conditioning, enhancing their digestion. On the other hand, conventional operations such as chemical conditioning and dewatering of the activated sludge can be substantially enhanced by fungal conditioning. This is reasonably possible because the belt press typically produces a dewatered cake containing $18 \%$ to $25 \%$ of solids when treating a typical combination of primary and secondary sludge, and many plants produce a cake containing $15 \%$ to $18 \%$ of solids when dewatering anaerobically digested material [5]. In this study, fungal conditioning produces a sludge cake containing $16 \%$ of solids. This value is closed to those mentioned before. On the other hand, a recessed-plate filter press with chemical conditioning of primary and secondary sludge can only achieve dewatered cakes with not more than $50 \%$ of solids content [5]. This dewatering capacity could be enhanced by the fungal conditioning, or even chemical conditioning could be reduced or eliminated, improving the quality of the sludge or biosolids generated because eliminating chemicals consequently could facilitate its disposal. Furthermore, the use of fungal conditioning could contribute to reducing the volume of the activated sludge which could consequently reduce the costs of management and disposal. The energy required to operate the dewatering equipment could also potentially be reduced because the fungal conditioning produces a sludge that is easier to dewater.

Even though the results of this research are promising, more research is needed to evaluate the effect of fungal dosing (in this study, a fixed dosage was used) and the effectiveness of the T. reesei and A. oryzae in other types of sludge or biosolids, for instance primary sludge or biosolids from sludge digestion processes. The potential effects of the sludge properties, especially volatile suspended solids (VSS), on fungal dewaterability must be investigated when prior sludge treatment (by mechanical thickening or anaerobic digestion) is conducted because the solids content, VSS, and EPS concentration of activated sludge (before treatment) and biosolids (after digestion) are different. Consequently, fungal dosing and retention times of the fungal conditioning used in this study could not be applicable. The performance of a belt or filter press with fungal-conditioned sludge must be evaluated to determine the benefits of the fungal conditioning. Furthermore, comparisons of our proposed method with other techniques for thickening, conditioning and dewatering in terms of carbon and energy footprints should be conducted to elucidate the potential of fungal conditioning in practical applications. It is important to remark that the scope of this study was the evaluation of fungal conditioning on the dewaterability of the activated sludge, rather than the assessment of the performance of the dewatering operations and equipment.

\section{Conclusions}

Two fungi (T. reesei and A. oryzae) were used as additives to enhance the dewaterability of activated sludge obtained from a municipal wastewater treatment plant. Fungal conditioning increased the solids content of the sludge cake and reduced the capillary suction time, denoting the enhancement of sludge dewaterability. Hydrolysis of EPS by extracellular hydrolytic enzymes secreted by fungi eased the release of water from the flocs structure of sludge and consequently enhanced its dewaterability. The decay (degradation) of biomass did not contribute to enhancing the sludge dewaterability. During the hydrolysis of bound and soluble EPS, proteins and carbohydrates were released from the polymer chains. Simple carbohydrates were used by the fungal and microbial communities to increase their population. Fungal conditioning had a positive effect on the microbial community. Furthermore, the increase of the fungal population augmented the hydrolytic activity of enzymes over the sludge EPS, enhancing the water release and, consequently, the dewaterability of the activated sludge. From a practical point of view, the results of this study are promising because conventional thickening, chemical conditioning and dewatering operations could be improved by a fungal conditioning process, obtaining important benefits in terms of less energy consumption and greater quality of sludge or 
biosolids (because chemicals could be eliminated), which could facilitate their disposal and finally reduce the costs of treatment and disposal.

Acknowledgments: This work was supported by the National Council of Science and Technology (CONACYT) of Mexico and the Tecnológico de Monterrey.

Author Contributions: Miguel Ángel López Zavala obtained the funds to conduct the research. Furthermore, he conceived and designed the experimental procedure, analyzed and discussed the results, prepared tables and figures, and wrote the paper. Jorge Isidro Ramos Patlán conducted all the experiments and analyzed the raw data.

Conflicts of Interest: The authors declare no conflict of interest. The founding sponsors had no role in the design of the study; in the collection, analyses, or interpretation of data; in the writing of the manuscript; or in the decision to publish the results.

\section{References}

1. Neyens, E.; Baeyens, J.; Dewil, R.; De Heyder, B. Advanced sludge treatment affects extracellular polymeric substances to improve activated sludge dewatering. J. Hazard. Mater. 2004, 106, 83-92. [CrossRef]

2. Egemen, E.; Corpening, J.; Nirmalakhandan, N. Evaluation of an ozonation system for reduced waste sludge generation. Water Sci. Technol. 2001, 44, 445-452. [PubMed]

3. Conagua. Diagnóstico del Programa U031 "Operación y Mantenimiento de Las Plantas de Tratamiento de Aguas Residuales"; Publication of the Ministry of Environment and Natural Resources (SEMARNAT): Mexico City, Mexico, 2014. (In Spanish)

4. Guo, J.S.; Xu, Y.F. Review of Enzymatic Sludge Hydrolysis. J. Bioremed. Biodegrad. 2011, 2. [CrossRef]

5. American Society of Civil Engineers (ASCE); Environmental and Water Resources Institute (EWRI); Water Environment Federation (WEF). Vol. 3: Solids processing and management. In Design of Municipal Wastewater Treatment Plants, 5th ed.; McGraw Hill: New York, NY, USA, 2009.

6. Tchobanoglous, G.; Burton, F.L.; Stensel, H.D. Wastewater Engineering, Treatment and Reuse, 4th ed.; McGraw Hill: New York, NY, USA, 2003.

7. Chang, G.R.; Liu, J.C.; Lee, D.J. Co-conditioning and dewatering of chemical sludge and waste activated sludge. Water Res. 2001, 35, 786-794. [CrossRef]

8. Zheng, G.; Wang, Z.; Wang, D.; Zhou, L. Enhancement of sludge dewaterability by sequential inoculation of filamentous fungus Mucor. circinelloides ZG-3 and Acidithiobacillus. ferrooxidans LX5. Chem. Eng. J. 2016, 284, 216-223. [CrossRef]

9. Murugesan, K.; Selvam, A.; Wong, J.W.C. Flocculation and dewaterability of chemically enhanced primary treatment sludge by bioaugmentation with filamentous fungi. Bioresource Technol. 2014, 168, 198-203.

10. Molla, A.H.; Fakhru'l-Razi, A. Mycoremediation-A prospective environmental friendly technique of bioseparation and dewatering of domestic wastewater sludge. Environ. Sci. Pollut. Res. 2012, 19, 1612-1619. [CrossRef] [PubMed]

11. Raghukumar, C.; D'Souza-Ticlo, D.; Verma, A. Treatment of colored effluents with lignin-degrading enzymes: An emerging role of marine-derived fungi. Crit. Rev. Microbiol. 2008, 34, 189-206. [CrossRef] [PubMed]

12. Alam, M.Z.; Fakhru'l-Razi, A.; Molla, A.H. Biosolids accumulation and biodegradation of domestic wastewater treatment plant sludge by developed liquid state bioconversion process using batch fermenter. Water Res. 2003, 37, 3569-3578. [CrossRef]

13. Alam, M.Z.; Fakhru'l-Razi, A. Enhanced settleability and dewaterability of fungal treated domestic wastewater sludge by liquid state bioconversion process. Water Res. 2003, 37, 1118-1124. [CrossRef]

14. Fakhru'l-Razi, A.; Molla, A.H. Enhancement of bioseparation and dewaterability of domestic wastewater sludge by fungal treated dewatered sludge. J. Hazard. Mater. 2007, 147, 350-356. [CrossRef] [PubMed]

15. Chancharoonpong, C.; Hsieh, P.C.; Sheu, S.C. Enzyme production and growth of Aspergillus oryzae S. on Soybean Koji Fermentation. APCBEE Procedia 2012, 2, 57-61. [CrossRef]

16. Machida, M.; Asai, K.; Sano, M.; Tanaka, T.; Kumagai, T.; Terai, G.; Kusumoto, K.I.; Arima, T.; Akita, O.; Kashiwagi, Y.; et al. Genome sequencing and analysis of Aspergillus oryzae. Nature 2005, 43, 1157-1161. [CrossRef] [PubMed]

17. Martinez, D.; Berka, R.M.; Henrissat, B.; Saloheimo, M.; et al. Genome sequencing and analysis of the biomass-degrading fungus Trichoderma. reesei (syn. Hypocrea jecorina). Nat. Biotechnol. 2008, 26, 553-560. [CrossRef] [PubMed] 
18. American Type Culture Collection: Manassas (ATCC). Mycology Culture Guide Tips and Techniques for Culturing Yeasts and Filamentous Fungi; ATCC: Manassas, VA, USA, 2013.

19. American Public Health Association (APHA); American Water Works Association (AWWA); Water Environment Federation (WEF). Standard Methods for the Examination of Water \& Wastewater, 20th ed.; American Public Health Association: Washington, DC, USA, 2005.

20. Frolund, B.; Palmgren, R.; Keiding, K.; Nielsen, P.H. Extraction of Extracellular Polymers from Activated Sludge Using a Cation Exchange Resin. Water Res. 1996, 30, 1749-1758. [CrossRef]

21. Peterson, G.L. A simplification of the protein assay method of lowry et al. which is more generally applicable. Anal. Biochem. 1977, 83, 346-356. [CrossRef]

22. Scholz, M. Review of recent trends in capillary suction time (CST) dewaterability testing research. Ind. Eng. Chem. Res. 2005, 44, 8157-8163. [CrossRef]

23. Sawalha, O.; Scholz, M. Modeling the relationship between capillary suction time and specific resistance to filtration. J. Environ. Eng. ASCE 2010, 136, 983-991. [CrossRef]

24. Chen, G.W.; Lin, W.W.; Lee, D.J. Capillary Suction Time (CST) as a Measure of Sludge Dewaterability. Water Sci. Technol. 1996, 34, 443-448.

25. Merrylin, J.; Kaliappan, S.; Adish Kumar, S.; Yeom, I.T.; Rajesh Banu, J. Enhancing aerobic digestion potential of municipal waste-activated sludge through removal of extracellular polymeric substance. Environ. Sci. Pollut. Res. 2014, 21, 1112-1123. [CrossRef] [PubMed]

26. Adav, S.; Chao, L.; Sze, S. Quantitative secretomic analysis of Trichoderma reesei strains reveals enzymatic composition for lignocellulosic biomass degradation. Mol. Cell. Proteom. 2012, 11. [CrossRef]

27. Chutmanop, J.; Chuihulcherm, S.; Chisti, Y.; Srinophakun, P. Protease production by Aspergillus oryzae in solid-state fermentation using agroindustrial substrates. J. Chem. Technol. Biotechnol. 2008, 83, 1012-1018. [CrossRef]

(C) 2016 by the authors; licensee MDPI, Basel, Switzerland. This article is an open access article distributed under the terms and conditions of the Creative Commons Attribution (CC-BY) license (http://creativecommons.org/licenses/by/4.0/). 